\title{
An Investigation of Learning Analytics in an Online Video-based Learning Platform
}

\author{
Ethan Hoe Chuan Sim \\ Department of Computing and Information Systems \\ School of Science and Technology \\ Sunway University, Malaysia \\ Email:14030571@imail.sunway.edu.my
}

\author{
Sian Lun Lau \\ Department of Computing and Information Systems \\ School of Science and Technology \\ Sunway University, Malaysia \\ Email: sianlunl@sunway.edu.my
}

\begin{abstract}
Using an online platform as a teaching and learning tool has been more common since the last decade. An advantage of this approach is the ability to capture implicit information, and hence learning analytics may be implemented to assist improvement in learning experience and efficiency. Thus, this paper aims to investigate and identify what features are needed by stakeholders to be included in an online video-based learning platform in order to implement learning analytics. A prototype learning platform has been developed. It has been evaluated through a survey by a group of testers to obtain feedback and review regarding their learning and usage experience. The majority of the participants of the survey have shown interest in the features implemented by the system and would like to see these features implemented in a working video learning platform.
\end{abstract}

\section{Introduction}

E-learning has been an active research area for decades. In the last decade, with the emergence of big data analytics, learning analytics has been one of the leading development areas in e-learning [1]. With the popularity of online video platforms (such as Youtube and Vimeo) to a modern online learning platform (such as Coursera, Udemy and Udacity), video-based learning is ade popular and common. Such developments also enable the opportunity to capture implicit user interaction through learning video and open up the ability to perform learning analytics [2]. An online video learning platform with data analytics functions applies implicit data acquisition through user interaction and produces visual representations of data as well as provides suggestions for areas of study.

E-learning is a form of imparting knowledge through computers. Such knowledge can be acquired through different mediums such as e-books, presentation slides, lecture notes, voiced recorded lectures and even video lectures. Learning through videos have shown to have many beneficial factors as an effective teaching method. People may learn better through sound and sight, and videos tend to allow the audience to engage better in the topics through illustrations supported by voice commentary [3].

However, it also comes to mind that not everyone prefers to use video learning as a source of knowledge. The reasons for this are mainly about the videos being confusing for some audiences. They might lack context, which depends on the knowledge that is required to understand that specific topic (pre-requisites). Moreover, due to the lack of immediate feedback or engagement from the lecturers, students might find it challenging to get their questions answered at that given point in time. Other factors such as the video being uninteresting and non-interactive may be taken into consideration when dealing with the dissatisfaction of students when learning through videos. Though all of these reasons being the case, students also find it challenging to keep track of where the sub-topics lie in the video that they are watching and do not know which parts they might have misunderstood due to it. Thus, the project proposed has the purpose of tackling some of the mentioned issues that students face.

In this paper, a web-based online video learning platform has been designed and developed as a prototype learning platform that aims to provide learning analytics. This platform was created as a proof of concept system to study whether visualisation obtained through data analytics could improve one's perception and experience in using a video learning platform to acquire new knowledge. The data captured through usage of learners for a lesson can be analysed, summarised and visualised for both learners and lecturers. In this way, learners will see which areas of study they tend to focus on while getting suggestions on which areas they should be looking at.

The structure of this paper is as follow: Section 2 presents a review of related work in this area. This is followed by Section 3 where the methodology and implementation are presented. Section 4 discusses the outcome and findings before the paper is concluded in Section 5.

\section{Related work}

\subsection{E-learning Platforms}

There exist many online learning platforms in the last decade. With the rise of the Massive Open Online Courses (MOOC) platforms, anyone with decent Internet connectivity can now take up classes, sometimes even at no cost, 
at own convenience and comfort. This subsection presents two selected leading platforms to understand how these platforms have implemented their layout and design that may affect the learning experience.

Udacity [4] is an online learning platform that began as an experiment to provide knowledge to people anywhere. It started with free courses, but currently, it offers also paid contents to allow learners to access courses through their online platform. The user interface is simple and straight forward. Clear instructions are presented so that learners can navigate through the lessons with ease. Overall, learners can be engaged in a learning experience that is welcoming. The organisation of the contents and the sub-topics are wellplanned. Visual cues were used to indicate progress which is again good practice for modern UI/UX design.

edX [5] is an online platform for education and learning founded by Harvard University and Massachusetts Institute of Technology (MIT). The layout is pretty standard compared to other current providers, so learners should be familiar with navigation without much trouble. The categorisation is more extensive than other services. Hence, it may be more complicated for a new learner to find the desired contents. It also means that it is more likely to find a more specific content if one can use the right keywords to filter. The strength of the platform is the support for a student to ask questions with other learners at a certain point during a lesson. It also provides a progression page to help users to track their learning progress. In this way, a learner may analyse how he could improve and understand his mistakes better.

Other platforms may have different course offerings or business models. However, from the learning experience and layout design point of view, they do not differ significantly from the two examples above.

\subsection{Video Media Platforms Features}

Since the focus of the platform will be revolving around video lectures, reviewing the features of a video media platform provides a significant impact on how users interact with it. Many of the existing online learning platforms use video playback and control interfaces as well as approaches identical to these popular video media platforms.

Youtube [6] will be perhaps the most popular online video media platform for almost two decades ago. The platform allows users to upload, rate, share and comment on videos. Besides that, users can follow other users to keep updated on videos released. Thus, Youtube is now considered a social media platform instead of a media play platform. What is interesting for this project is the video analytics feature offered by Youtube as the capability to analyse interaction and view behaviour may be useful for learning analytics too. The users can not only interact via comments but also can indicate the position of the playback (timestamp) to pinpoint where their interests and comment are about precisely. It also has a video suggestion where machine learning is used to recommend users contents they may like and wish to explore.
Another popular online video platform will be Netflix [7]. It was founded in 1997 first as an online media streaming service provider through subscription. Today, Netflix is also a production house with its film and series productions. The media suggestion feature is similar to many other online media platforms, including Youtube. Navigation wise, Netflix will not be too different from other comparable platforms. However, there is no comment feature on Netflix.

\subsection{Platform Features to enable Data Collection, Analysis and Visualisation}

There are existing services and techniques that aim to explore different ways of user activity tracking on online platforms. Similarly, the tracked information will be useful for further analysis that may then be used to improve learning experience and efficiency. The following subsections elaborate some of these techniques:

2.3.1. Click and Interaction Tracking. In Google Analytics API [8], the creation of analytic events rely heavily on click tracking, which is the tracking of user activity through mouse clicks. The API itself will track action done by the user depending on the category. With the label and value, which are included as part of the optional fields of tracking, the web object that is being interacted with will be recorded for analysis. The interaction among learners and between learners and instructors may also give hints to help to understand and improving learning experience [9]. This technique will be important as there will not be face-to-face physical interactions like in a conventional learning environment. As learning is distribution, likely mobile and asynchronous, the tracking of any learning and online interactive activities will be the primary input for learning analytics.

2.3.2. Location Tracking. With most companies that have big data analytics, one of the permissions they commonly ask for from the user would be the access to the user's location. Learning analytics can also benefit from location tracking if the location information may help both learners and teachers to improve learning experience and efficiency. One good example will be learning applications through mobile-based serious game approach [10]. Knowing the where-about of the learning may help to adapt the design of delivery to motivate and encourage learning not restricted to a fixed location.

2.3.3. Visualisation through Charts and Graphs. Though being more traditional methods, the data collected can be shown through a chart or graph. This kind of representation is often used with areas that are more statistical and involve more mathematics to provide conclusions to the data. The visualisation will support instructors to be able to gain insights and understanding of how the learners' behaviour may be linked to the learning experience and efficiency concerning the pedagogical review and improvement [11]. 
2.3.4. Heat Maps. Heat maps come in a variety of different ways of portraying user activity. This visual representation focuses on highlighting areas on a web page that users interact with. The data would be represented as heat signatures on a copy of the web page's layout that pinpoints hot zones on the website. A service like Mouseflow [12] offers a variety of heat maps, each having their own purpose. Heat maps through the mouse pointer, scroll section of the page, and even through geographical placement has been provided by Mouseflow. Another work that uses a heat map approach to reflect and review learners behaviour is the Moodle Activity Viewer [13]. By understanding which elements in a learning platform learners are and aren't more frequently accessed, it may help to reflect ways to improve learning and interaction when such information can be made available.

\subsection{Comparison and Analysis}

Based on the above review and analysis, the prototype platform should focus on the following points:

2.4.1. General Platform Layout. The first impression given to any learner is rather important, so the general platform layout should be simple, modern and also clean. The items on the pages should not be cluttered but should be tidy and organised. While this is not something very new, but it will continue to be an important factor not to be neglected.

2.4.2. Catalogue Layout and Features. By reviewing how both Udacity and edX manage and organise their courses and contents, search and filter functions will be an essential feature for a learning platform. The platform should use a catalogue system that allows learners to search and filter in order to find the desired contents within the shortest time.

2.4.3. Lesson Progress Visualisation. Through user progress and activity, some necessary information can be visualised for both learners to support and motivate them to learn better. For the instructors, the visualisation will not only help the management of the delivery but also pedagogical review and improvement.

2.4.4. Comments and Discussion. Looking at how the line between video platform and social media have been blurred, the comment and discussion feature should be incorporated into a modern video learning platform. Not only one gets to allow more interactions among users of the platform (both learners and instructors), but the implicit information left behind through these comments and discussions is another rich source of information for potential analytics.

\section{Methodology}

The project was carried out in three main phases. In phase 1 , the researchers interviewed users regarding their acceptance and respective reasons for video-based learning.
The outcome helps the former to design on requirements for the design and implementation of the platform, which is phase 2 of the project. In phase 3, user testing was carried out to gather user feedback on the implemented platform.

\subsection{Learners perception of Video-based Learning}

Eight users were invited for an interview for the requirement gathering. $12.5 \%$ of the interviewees indicated that they do not like learning through videos. Four out of eight responded positively, while the rest are on the fence. When it comes to why they would opt for learning through videos, many stated convenience and possibility to self-pace as main factors. As compared to using purely electronic notes or presentation slides documents, video-based materials are seen as more engaging and extensive. The few who have a more negative view on this matter stated that the lack of interactive nature and uninteresting implementation of the recorded videos are the major drawbacks.

The comments and feedback have one element in common - learners are expecting interaction and engagement in their learning experience, not just the contents. This will continue to be a factor asynchronous online delivery (using pre-recorded videos) will face. While this is an important factor, this project will not be able to address this and will keep this as a possible future extension. Being able to allow learners to view and review the contents will be one of the main features of the platform, and this will also be a feature that can be used for learning analytics.

\subsection{Platform Design and Components}

As a result from the review on existing video platforms as well as the feedback from the interviewees, the following functions have been selected:

1) Video Playback Control Functions

- Play/Pause Function

- Playback control of a video

- Video Progression Tracking

- Progress Bar Functions - Show and allow choosing location of time in videos

- Current Time/Duration Calculations Show the time progress and duration

- Volume Control

- Muting Function - Mute the video

- Volume Bar Function - Control the loudness of the video.

- Full Screen Toggle

- Toggle full screen view of a video

2) Comments-Discussion Functions

- Comment Function 
- Allow users to post a comment

- Replying Function

- Allow users to comment on existing comments

- Categorization Function

- Specifying which topic the comment is under

3) Data Acquisition and Analysis Functions

- Implicit Data Acquisition

- User interaction and playback generated data will be recorded for analysis purpose

- Data Analysis

- Data Mapping and Modelling

* Mapping data retrieved to their respective areas.

- Data Interpretation

* Providing "meaning" or context to the data mapped or modelled.

4) Data Visualisation Functions

- After mapping and evaluating the data collected, the data is then organised and Visualised through charts and graphs

5) Website Design and Architecture

- Dynamic Communication and Responses between Client and Server

- Functions such as Requests to Server and Retrieve Data from Server help in the placement of web objects through a Web Object Loader Function. This provides a dynamic behaviour to the platform without having to reload existing components in the page.

- Responsive Design

- To allow users to access the platform through mobile means, the website was created to be responsive to mobile screen sizes. Functions such as Resize Object Functions and Object Ratio Calculations will be used to allow all web objects to be fitted into a mobile form factor.

- Database for Storage

- Data queries will be enabled to allow insertion and manipulation of data at the backend

- Backend Functions

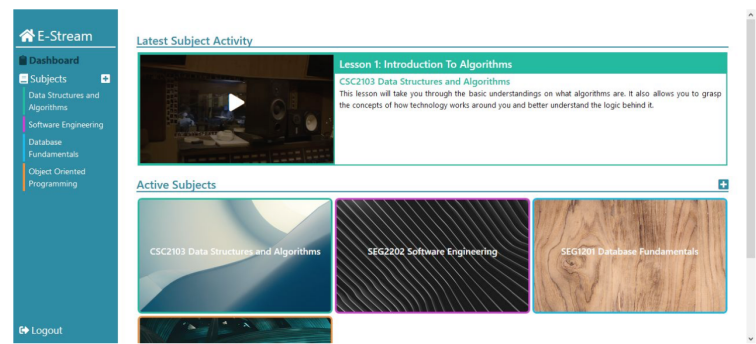

Figure 1. Dashboard Screen - Desktop View

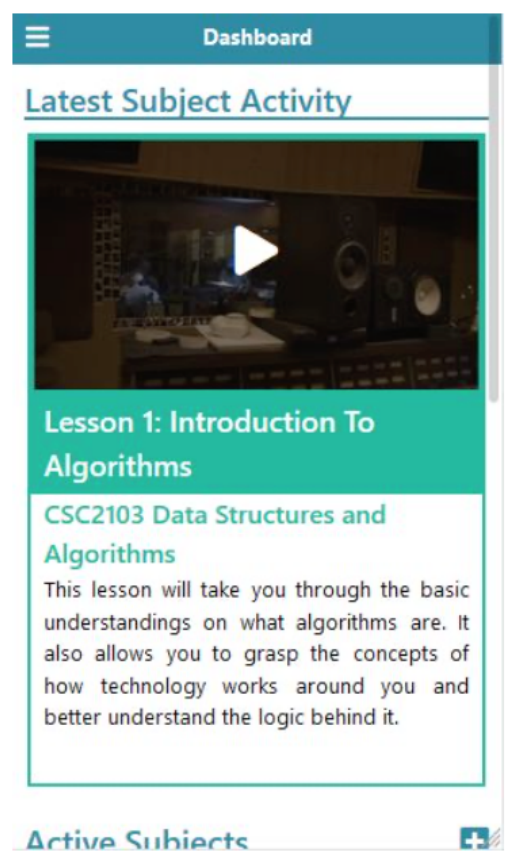

Figure 2. Dashboard Screen - Mobile View

- Functionalities for the back end would include Web Object Creation that allows elements in the HTML code to be precreated to be pushed out to the front end.

Functions 1 and 2 are rather common functions one would find in almost all online video platform. Similarly, functions 5 are also pretty standard and common in most modern web platforms. The most important and distinct functions that will answer to the goal of the implementation and study will be on functions 3 and 4 .

The end product is a prototype Video-Learning platform built using modern libraries and framework, such as Bootstrap $^{1}$, Chart.js ${ }^{2}$, Font Awesome Toolkit ${ }^{3}$, together with HTML + CSS + Javascript for frontend development. As for the backend, the platform uses PHP and MariaDB.

Example look and feel are depicted in Figure 1 and 2. These figures show the default view when a student enters

1. https://getbootstrap.com/, last visited on 18th September 2020

2. https://www.chartjs.org/, last visited on 18th September 2020

3. https://fontawesome.com/, last visited on 18th September 2020 


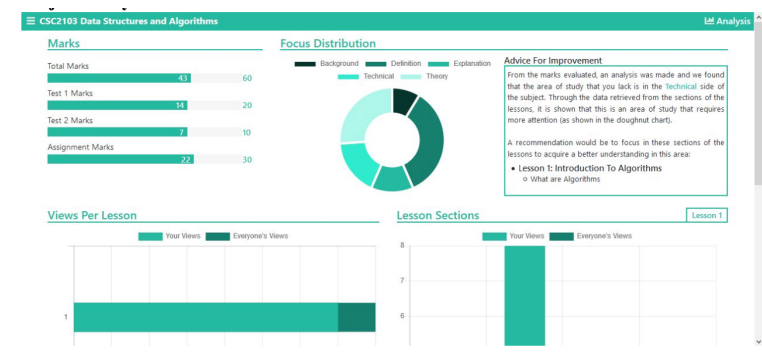

Figure 3. Subject Analytics Screen - Lecturer's View \#1

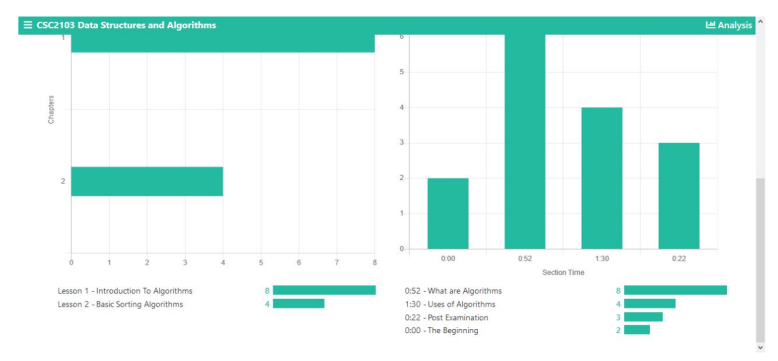

Figure 4. Subject Analytics Screen - Lecturer's View \#2

into the system. From here, one can then enter input a subject and begin learning by watching the video contents uploaded and designed by a lecturer. As lecturers, they will be interested not just to upload and prepare lessons using this platform. The platform provides the ability to display the visualisation of the different aspects of lessons in a subject. As shown in Figure 3 and 4, The subject analytics screen displays information such as marks distribution, focus distribution and also lesson view statistics.

Worth noting is the feature to allow learners to compare their views with the rest of the class as well as to see view break downs according to sections within a single video of a lesson. Such a feature will indirectly encourage a learner to compare and benchmark his progress with the rest of the class. View per Section (see Figure 5) will also be a helpful feature as it not only help learners to "compete" with one another on making progress. Sections within a lesson that have gained a higher number of views may also indicate either a section is difficult to understand or it is of a certain level of importance.

\subsection{User Testing}

A simple user testing evaluation was carried out to understand whether a user will require significant time and effort to navigate and use the platform. Seven testers were engaged to carry out the following tasks:

1) Login using a given user id and password

2) Access the subject catalogue through the dashboard

3) Search for Data in the search bar

4) Use filter to narrow down the search through a given subject code

5) Enrol into the given subject as a learner

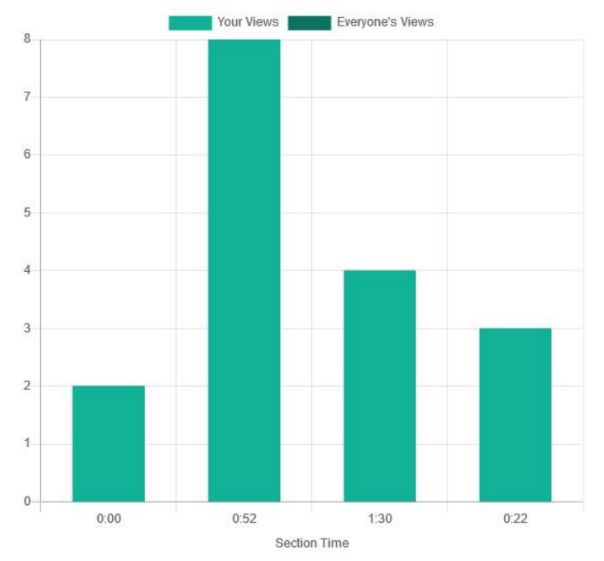

Figure 5. Views per Section in a Lesson

6) Go to the subject screen

7) Play a video of the uploaded lessons in this subject

8) Go to the analytics screen

9) Read the suggestion provided in the Advice for Improvement section

Generally, all testers can complete the given tasks within 5 minutes. The expected average time for one to $\log$ in and navigate to start a lesson would have been around 34 minutes. Besides this, the testers are also encouraged to explore the platform after completing the given tasks. Upon completion of the exploration, they were given a survey to obtain their feedback on the platform. Details of the posttesting survey will be presented in the next section.

\section{Evaluation and Discussion on the User Sur- vey and Feedback}

The survey aims to get the users impression and perception of the prototype platform. The questions are as follow:

1) What words would you use to describe about the layout of the Dashboard? (select one or more answers)

2) What words would you use to describe about the layout of the Subject Catalogue Screen? (select one or more answers)

3) How was the layout of the information from the results provided by the search function?

4) What words would you use to describe about the layout of the Subject Profile Screen? (select one or more answers)

5) What words would you use to describe about the layout of the Lesson Screen? (select one or more answers)

6) What words would you use to describe about the layout of the Subject Analytics Screen? (select one or more answers)

7) what words would you use to describe about user interface of the platform? (select one or more answers) 
From all seven questions, The selected descriptions selected by the testers after exploring the platform are Clean, Organised, Professional and Appealing. Among them, Clean and Organised are the highest selected descriptions. From Question 7, testers rated the user interface as follow:

- Organised $(100 \%)$

- Appealing (85.7\%)

- Professional $(85.7 \%)$

- Clean $(57.1 \%)$

Overall, testers found the platform organised, appealing and professional. No negative descriptions were selected. The general impression is somewhat positive, giving a hint that the user experience and interface need to be emphasised in order to encourage usage.

When the survey further asked the testers on which screen they like in terms of layout and design, Subject Analytics Screen got the highest vote $(100 \%)$ followed by Dashboard, Lesson and Subject Profile (71.4\%). This is also a positive outcome as the goal of the design and implementation of the platform was to provide the analytics capability to enhance teaching and learning using the video learning approach.

The final two questions in the survey investigate users' experience and their view on how the design and features may contribute to a better experience. Majority rated 8 or higher out of 10 for the experience they had after the test. Similarly, the testers also confirmed that the design and features of the platform contribute to a better learning experience. Based on the previous feedback, the learning experience is not limited to only the content and its delivery. The appeal, organisation as well as look and feel of the platform are also factors contributing to good learning experience.

As the goal is to explore and investigate the acceptance and usefulness of the analytics ability on a video learning platform, the feedback justifies the need not only to capture usage and control of learning contents, but also to visualise progress as well as comparison with peers to motivate and encourage learning. On the other hand, the visualisation also help the lecturer to gain insights into the progress and effectiveness of a lesson.

\section{Conclusion}

The project's main objectives were to understand factors that may improve the learning experience of students through analytics as well as features that improve the user experience for an online video learning platform. For this purpose, a prototype platform has been developed and tested by volunteer testers. The feedback from the testers was generally positive and gave good insights into ways to improve learning and user experience for an online video learning platform. The video section feature and display of analytics for both learners and lecturers are seen as good features to improve the intended experience.

As possible next step, the platform can be extended with machine learning-enhanced analytics to pro-actively monitor and analyse performance and learning efficiency of a learner. Another future will be obtaining a larger number of testers to validate the feasibility and usability of the platform for large scale roll out. The collected data through larger group of users will also assist improvement of the desired analytics research.

\section{Acknowledgment}

The authors would like to thank the volunteers who have participated in the evaluation of the prototype system and provide valuable feedback.

\section{References}

[1] R. Bodily and K. Verbert, "Review of research on student-facing learning analytics dashboards and educational recommender systems," IEEE Transactions on Learning Technologies, vol. 10, no. 4, pp. 405418, 2017.

[2] M. N. Giannakos, D. G. Sampson, and Ł. Kidziński, "Introduction to smart learning analytics: foundations and developments in videobased learning," Smart Learning Environments, vol. 3, no. 1, pp. 1-9, 2016

[3] R. E. Mayer, "Using multimedia for e-learning," Journal of Computer Assisted Learning, vol. 33, no. 5, pp. 403-423, 2017.

[4] Udacity, "Udacity," 2020. Last accessed 16 September 2020.

[5] edX, “edx," 2020. Last accessed 16 September 2020.

[6] Youtube, "Youtube," 2020. Last accessed 16 September 2020

[7] Netflix, "Netflix," 2020. Last accessed 16 September 2020.

[8] G. Analytics, "Google analytics," 2020. Last accessed 16 September 2020

[9] N. R. Aljohani and H. C. Davis, "Significance of learning analytics in enhancing the mobile and pervasive learning environments," in 2012 Sixth International Conference on Next Generation Mobile Applications, Services and Technologies, pp. 70-74, 2012.

[10] V. M. Perez-Colado, D. C. Rotaru, M. Freire, I. Martinez-Ortiz, and B. Fernandez-Manjon, "Learning analytics for location-based serious games," in 2018 IEEE Global Engineering Education Conference (EDUCON), pp. 1192-1200, 2018.

[11] L. Corrin, G. Kennedy, P. De Barba, A. Bakharia, L. Lockyer, D. Gasevic, D. Williams, S. Dawson, S. Copeland, et al., "Loop: A learning analytics tool to provide teachers with useful data visualisations," in Australasian Society for Computers in Learning and Tertiary Education (ascilite2015, Perth: Ascilite, 2015.

[12] Mouseflow, "Mouseflow," 2020. Last accessed 16 September 2020.

[13] D. Clark, "The moodle activity viewer (mav) - heatmaps of student activity," 2013. Last accessed 16 September 2020. 